\title{
Perfil epidemiológico dos pacientes internados por acidente vascular cerebral no nordeste do Brasil
}

\author{
Epidemiological profile of patients hospitalized for stroke in northeastern Brazil \\ Perfil epidemiológico de pacientes hospitalizados por accidente cerebrovascular en el \\ noreste de Brasil
}

Anderson Matheus de Lima Barbosa ${ }^{1 *}$, Caio Coelho Machado Pereira ${ }^{1}$, João Pedro Rosal Miranda ${ }^{1}$, José Hamon de Lima Rodrigues ${ }^{1}$, José Roberto Oliveira de Carvalho ${ }^{1}$, Augusto César Evelin Rodrigues ${ }^{2}$.

\section{RESUMO}

Objetivo: Traçar um perfil epidemiológico dos pacientes com AVC no nordeste do Brasil no período de 2010 a 2019. Métodos: Tratou-se de um estudo retrospectivo, longitudinal, de caráter exploratório e descritivo, além de ser quantitativo. Este estudo se baseou nas informações contidas nos bancos de dados do Sistema de Informações Hospitalares (SIH) e no Departamento de Informática do Sistema único de Saúde (DATASUS) dos casos de AVC nos anos de 2010 a 2019. Resultados: Os resultados revelaram um aumento gradativo da doença durante 0 período analisado, ocorrendo uma discreta prevalência no gênero masculino $(50,8 \%)$. Quanto à mortalidade da doença, não houveram alterações bruscas no período estudado, observando-se uma média de $17,74 \%$ de mortes por casos. Conclusão: Constata-se, portanto, que é de fundamental importância o assunto abordado neste estudo tanto como fonte de informações epidemiológicas a respeito do AVC no nordeste do Brasil como sendo referência para outras pesquisas cientificas que abordem essa temática.

Palavras-chave: Acidente vascular cerebral, Estilo de vida, Perfil epidemiológico.

\section{ABSTRACT}

Objective: To draw an epidemiological profile of stroke patients in northeastern Brazil from 2010 to 2019. Methods: This was a retrospective, longitudinal, exploratory and descriptive study, in addition to being quantitative. This study was based on information contained in the databases of the Hospital Information System $(\mathrm{SIH})$ and in the Department of Informatics of the Unified Health System (DATASUS) of stroke cases in the years 2010 to 2019. Results: The results revealed an increase of the disease during the analyzed period, with a slight prevalence in males (50.8\%). Regarding the mortality of the disease, there were no sudden changes in the period studied, with an average of $17.74 \%$ of deaths per case. Conclusion: It appears, therefore, that the subject addressed in this study is of fundamental importance both as a source of epidemiological information on stroke in northeastern Brazil and as a reference for other scientific research that addresses this topic.

Keywords: Stroke, Lifestyle, Epidemiological profile.

\section{RESUMEN}

Objetivo: Trazar un perfil epidemiológico de los pacientes con ictus en el noreste de Brasil de 2010 a 2019. Métodos: Este fue un estudio retrospectivo, longitudinal, exploratorio y descriptivo, además de cuantitativo. Este estudio se basó en información contenida en las bases de datos del Sistema de Información Hospitalaria (SIH) y en el Departamento de Informática del Sistema Único de Salud (DATASUS) de casos de ictus en los años 2010 a 2019. Resultados: Los resultados revelaron un incremento de la enfermedad durante el período analizado, con una ligera prevalencia en el sexo masculino $(50,8 \%)$. En cuanto a la mortalidad de la enfermedad, no hubo cambios bruscos en el período estudiado, con un promedio de 17,74\% de muertes por caso. Conclusión: Parece, por tanto, que el tema abordado en este estudio es de fundamental importancia tanto como fuente de información epidemiológica sobre ictus en el noreste de Brasil como referencia para otras investigaciones científicas que abordan este tema.

Palabras clave: Accidente cerebrovascular, Estilo de vida, Perfil epidemiológico.

\footnotetext{
${ }^{1}$ Faculdade Integral Diferencial (UniFacid/Wyden). Teresina - PI. *E-mail: andersonbarbosa7570@outlook.com

${ }^{2}$ Centro Universitário Unifacid;]. Teresina - PI.
} 


\section{INTRODUÇÃO}

O Brasil, nos últimos anos, devido principalmente à evolução do capitalismo e da globalização que acarretam o aumento do sedentarismo e do consumo de "fast foods", tem passado por um período de transição epidemiológica. Período esse o qual doenças crônicas, como hipertensão, diabetes, obesidade e câncer, tem assumido o protagonismo no número de casos registrados, que antes era das doenças infecciosas. Nesse viés, percebe-se um aumento do risco de um crescimento de casos de acidente vascular cerebral ( $A \vee C)$, visto que a maioria desses distúrbios, como a hipertensão, a obesidade e o diabetes, estão intimamente relacionados a essa patologia (PIMENTEL VN e FILHA VAVS, 2019).

O AVC é uma doença que está relacionada à morte das células do cérebro de um indivíduo devido à ausência de um suprimento sanguíneo adequado a elas, o que proporciona uma carência de nutrientes e de oxigênio a essas células. Essa doença é dividida em 2 tipos principais: o acidente vascular cerebral isquêmico (AVCI), o qual pode ser classificado como lacunar, aterosclerótico ou embólico e o AVC hemorrágico (AVCH), que pode ser uma hemorragia intracerebral ou uma hemorragia subaracnóidea (NITRINI R, et al., 2015).

O AVCl é a principal causa de AVC no mundo, sendo caracterizado pela oclusão de uma artéria cerebral, o que leva à isquemia da área irrigada por essa artéria. Essa oclusão pode se dar de 2 maneiras principais: oclusão, devido à formação de uma placa aterosclerótica, que ocorre em decorrência do acúmulo de placas de gordura na parede vascular, evoluindo com a obstrução parcial ou total do lúmen do vaso, e oclusão devido a uma embolia, essa se caracteriza pela obstrução vascular por um corpo estranho que circule no sangue, sendo o tromboembolismo o quadro mais prevalente (ALVES HCBR, et al., 2016).

$\mathrm{O} A \mathrm{VCH}$, por sua vez, tem sua causa relacionada com a ruptura de um vaso no interior do parênquima cerebral, o que acarreta o extravasamento de sangue e, portanto, uma redução da efetividade perfusional. As principais causas dessa afeç̧ão são: hipertensão arterial, defeitos na coagulação, hemorragias subaracnóideas oriundas de malformações arteriovenosas e de roturas de aneurismas (MAMED SN, et al., 2019).

Dentre os fatores de risco relacionados ao AVC, a hipertensão arterial é o principal e o mais significativo. Uma elevada pressão arterial ocasiona, geralmente, microlesões no endotélio de vasos e capilares, o que contribui para o desenvolvimento de um AVC hemorrágico com extravasamento de sangue. Contudo, não é só a hipertensão que acarreta problemas. Se o indivíduo apresentar uma hipotensão arterial, e pode contribuir para que haja uma menor perfusão sanguínea em uma área isquêmica, gerando um aumento da área de acometimento do AVC. Assim, o ideal é que se obtenha um equilíbrio da pressão arterial (FURLAN NE, et al., 2018).

Segundo o Global Burden of Disease Study (2013), houve cerca de 25,7 milhões de sobreviventes de AVC, 6,5 milhões de óbitos por AVC e 10,3 milhões de novos AVC em todo o mundo. Apesar da redução da taxa de mortalidade dessa doença, ela ainda se faz muito presente (em valores absolutos) em todo o mundo e pode gerar, nos pacientes, sequelas e incapacidades as quais comprometem o estilo de vida do indivíduo para sempre (FERREIRA LE, et al., 2017). Isso é evidenciado no estudo realizado por Rolim AM, et al. (2020), o qual afirma que, segundo a Organização Mundial da Saúde, ocorrem cerca de 15 milhões de casos de AVC a cada ano no mundo, sendo que desses, 5 milhões resultam em morte e outros 5 milhões em incapacidades permanentes, acarretando, dessa maneira, considerável prejuízo à vida dos indivíduos acometidos por essa afecção.

O AVC, além de ser uma das principais causas de morte cardiovascular no mundo, gera incapacidade em um grande percentual dos pacientes e prejuízo aos cofres públicos (gastos com tratamento e reabilitação do indivíduo). Diante desse cenário, ressalta-se a importância da adoção de ações custo-efetivas que promovam uma redução da mortalidade e do percentual de indivíduos que evoluem com algum grau de incapacidade. Dentre esses fatores, tem-se o cuidado em unidades de AVC (previnem, detectam e tratam precocemente as complicações), as quais contribuem para um bom prognóstico da pessoa enferma, e a realizações de terapias de reperfusão, como a trombólise intravenosa e a trombectomia mecânica (RUIZ L, et al., 2020). 
Mediante o exposto, objetivou-se, com o estudo, identificar o perfil epidemiológico dos pacientes que sofreram acidentes vasculares cerebrais na região Nordeste do Brasil, priorizando os casos segundo sexo, faixa etária, área de maior prevalência e taxa de mortalidade.

Conhecer o perfil desses pacientes poderá favorecer a implantação de medidas que possam vir a diminuir o número de casos dessa patologia, que além das perdas em números de vidas ainda impactam economicamente nos serviços de saúde.

\section{MÉTODOS}

Este estudo abrangeu o perfil epidemiológico dos pacientes que adquiriram acidentes vasculares cerebrais (AVCs), na região Nordeste brasileira, no período de janeiro de 2010 a dezembro de 2019.

A pesquisa epidemiológica em questão mostrou-se como um estudo retrospectivo e longitudinal, com caráter exploratório e descritivo, ao mesmo tempo no qual é quantitativo. O trabalho foi posto em prática através do exame e interpretação das informações contidas nos bancos de dados do Sistema de Informações Hospitalares (SIH) do Departamento de Informática do Sistema Único de Saúde (DATASUS).

A população analisada deste estudo foi formada por todos os pacientes diagnosticados com AVC, de acordo com o código da Décima Revisão da Classificação Internacional de Doenças e Causas de Mortalidade (CID-10) para AVC, não especificado como isquêmico ou hemorrágico, que residiam na região nordeste do Brasil, no período delimitado pelo estudo e que foram contabilizados no formulário eletrônico do DATASUS.

Considerou-se como região nordeste do Brasil, as populações dos estados do Maranhão, Piauí, Ceará, Paraíba, Rio Grande do Norte, Pernambuco, Bahia, Alagoas e Sergipe. Entre as variáveis de interesse do estudo, foram selecionados, o sexo, faixa etária, raça/cor autodeclarada; taxa de mortalidade, internações hospitalares, média de permanência hospitalar e custo total das internações.

Somado a isso, foram buscadas referências bibliográficas em bancos/sites de pesquisas científicas, como o Scientific Electronic Library Online (Scielo), Business Source Complete (EBSCO) e o PubMed.

Para sintetizar os dados utilizou-se uma análise estatística descritiva e comparativa, através de números absolutos e relativos e proporção na base 100 e os resultados foram apresentados através de tabelas e gráficos, utilizando os aplicativos Microsoft Excel e Microsoft Word, agrupando os dados por filtro utilizado.

Não houve necessidade de envio do estudo ao CEP por se tratar de dados de Bancos públicos, mesmo assim, foram seguidas todas as recomendações éticas, emanadas pela Resolução № 466 do Conselho Nacional de Saúde.

\section{RESULTADOS}

O estudo analisou 386.453 casos de AVC ocorridos na região nordeste do Brasil, no período de 2010 a 2019.

Em relação às internações por $A V C$, durante os dez anos analisados, o número de internações ocasionadas por AVC, não especificado em isquêmico ou hemorrágico, de residentes na região Nordeste, foi de 386.453, sendo observado um aumento gradativo com o passar do tempo.

$\mathrm{O}$ ano de 2019 registrou o maior número de internações (47.924), representando um aumento de cerca de $60 \%$ em comparação ao primeiro ano de observação, 2010, que com 27.499 internações, foi o ano que apresentou o menor número de internações por essa causa, no período estudado (Gráfico 2).

O Gráfico 1 mostra as internações por AVC, segundo os estados, onde o que apresentou o maior número de internações durante o período foi o estado da Bahia com $113.142(29,27 \%)$ casos, seguido de Pernambuco com $81.022(20,96 \%)$ e do Ceará com 60.877 (15,75\%). 
Gráfico 1 - Internações segundo Unidade da Federação.

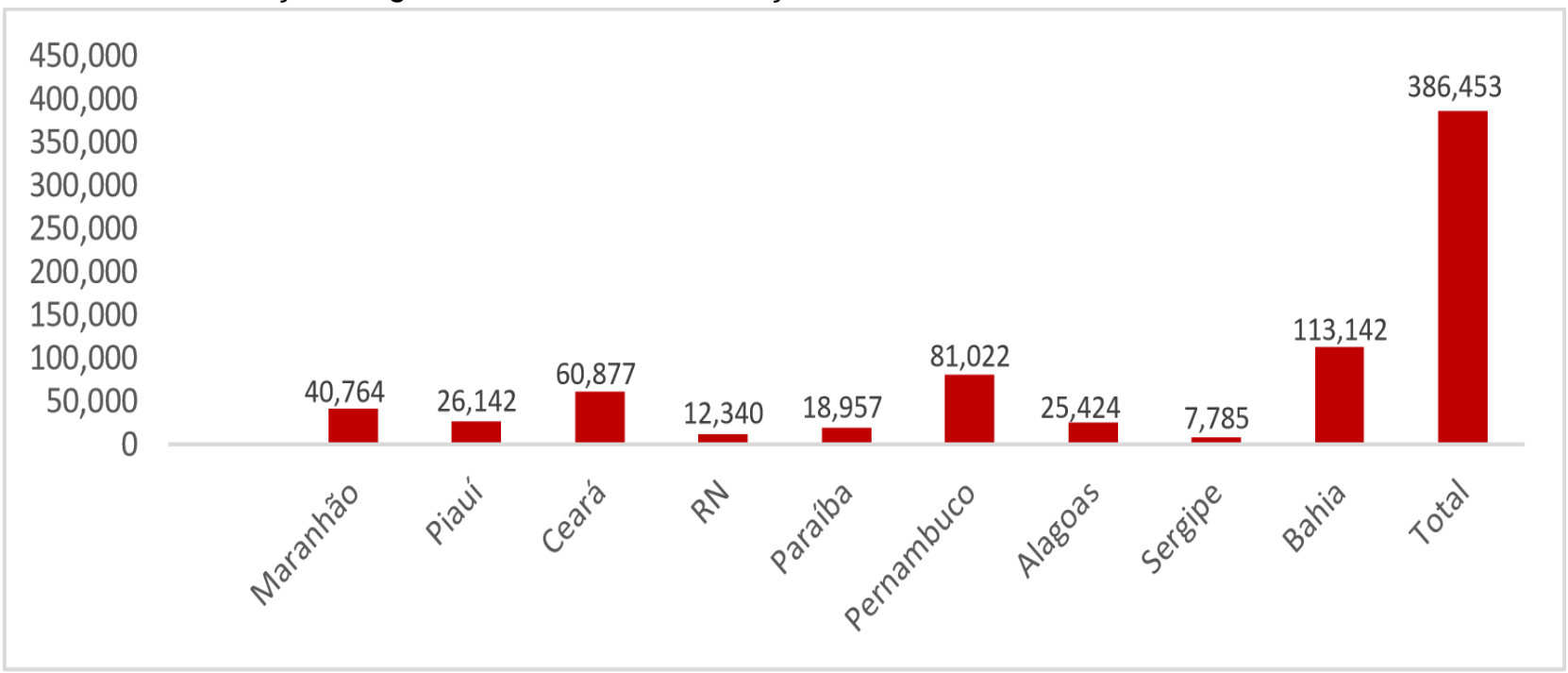

Fonte: Barbosa AML, et al., 2020. Dados extraídos do SIH/DATASUS.

Nesse sentido, percebe-se que os estados do Nordeste com maior número de internações são aqueles que possuem maior número de habitantes, de acordo com a estimativa populacional do Instituto Brasileiro de Geografia e Estatística, Bahia lidera com 14.873.064 habitantes, seguida de Pernambuco com 9.557.071 e do Ceará com 9.132.078. Além disso, o avanço das medidas diagnósticas possibilita que o número de casos relatados aumente (IBGE, 2019).

Para variável sexo, observou-se no Gráfico 2 um discreto predomínio do gênero masculino, com cerca de $50,8 \%$. Esses dados corroboram com Lima V, et al. (2006), que encontraram uma maior incidência de doenças cerebrovasculares em homens. O número de internações devido ao AVC aumentou gradativamente com o aumento da idade, sendo os maiores números observados na faixa etária acima de 60 anos. De maneira análoga, observa-se o que afirma Luna ACP, et al. (2013), a qual apresenta o AVC como uma doença que afeta predominantemente os idosos (Gráfico 2).

Gráfico 2 - Internações por sexo segundo faixa etária.

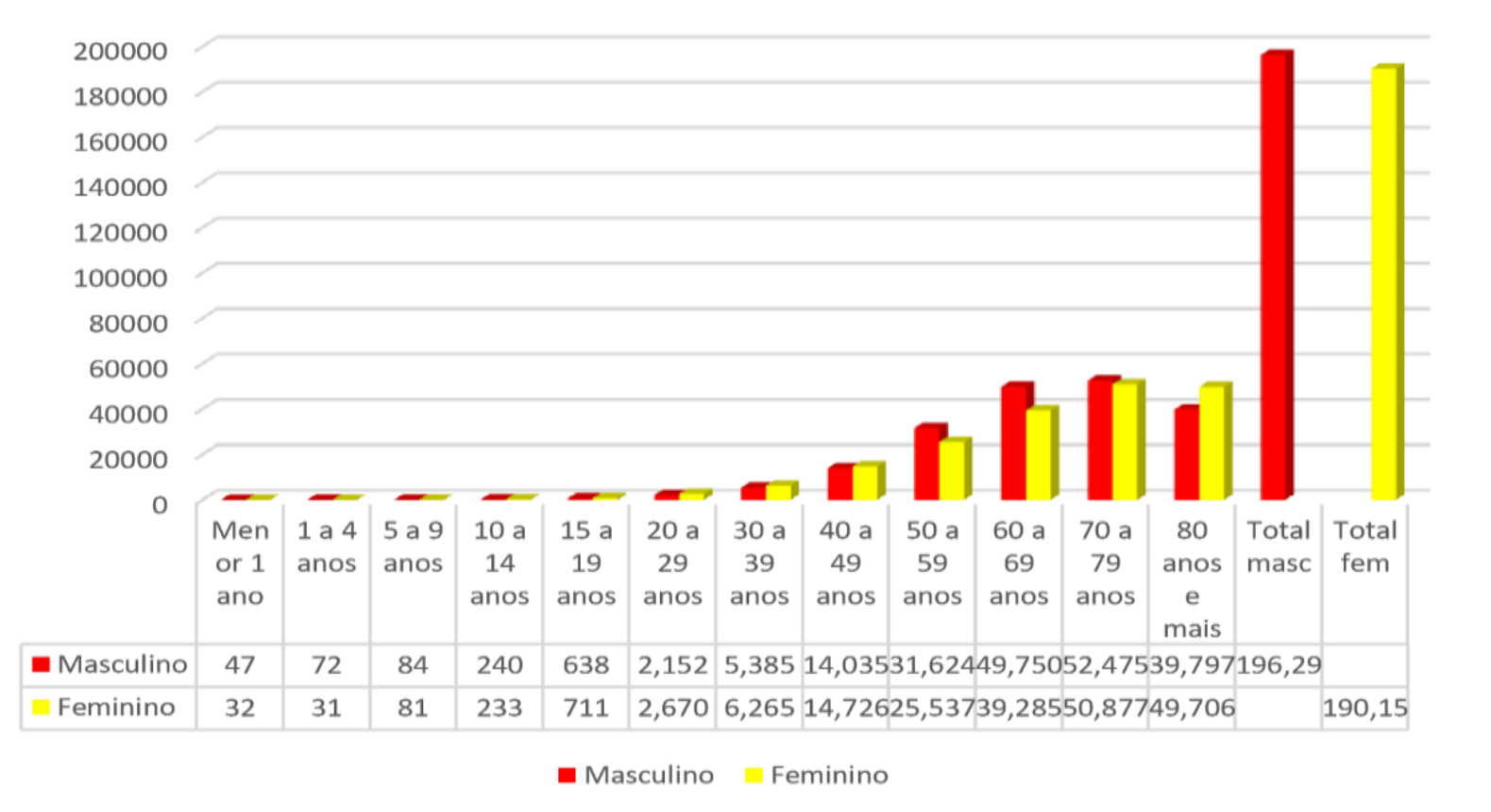

Fonte: Barbosa AML, et al., 2020. Dados extraídos do SIH/DATASUS. 
Ainda relacionado ao sexo, a classificação por idades demonstrou que no sexo masculino a maior prevalência da doença foi entre 70 e 79 anos, seguido da faixa etária de 60 a 69 anos e 80 anos ou mais. Já no sexo feminino, a maior prevalência também foi entre 70 e 79 anos, seguido pela faixa etária de 80 anos ou mais e 60 a 69 anos. Considerando-se os dois gêneros, a faixa etária com maior notificação de casos foi de 70 a 79 anos, sendo estes no total de 103.352 (26,74\%). Já o menor índice foi observado em pacientes menores de 1 ano, com apenas 79 casos (0,02\%) (Gráfico 3).

Gráfico 3 - Média de permanência hospitalar por sexo segundo faixa etária.

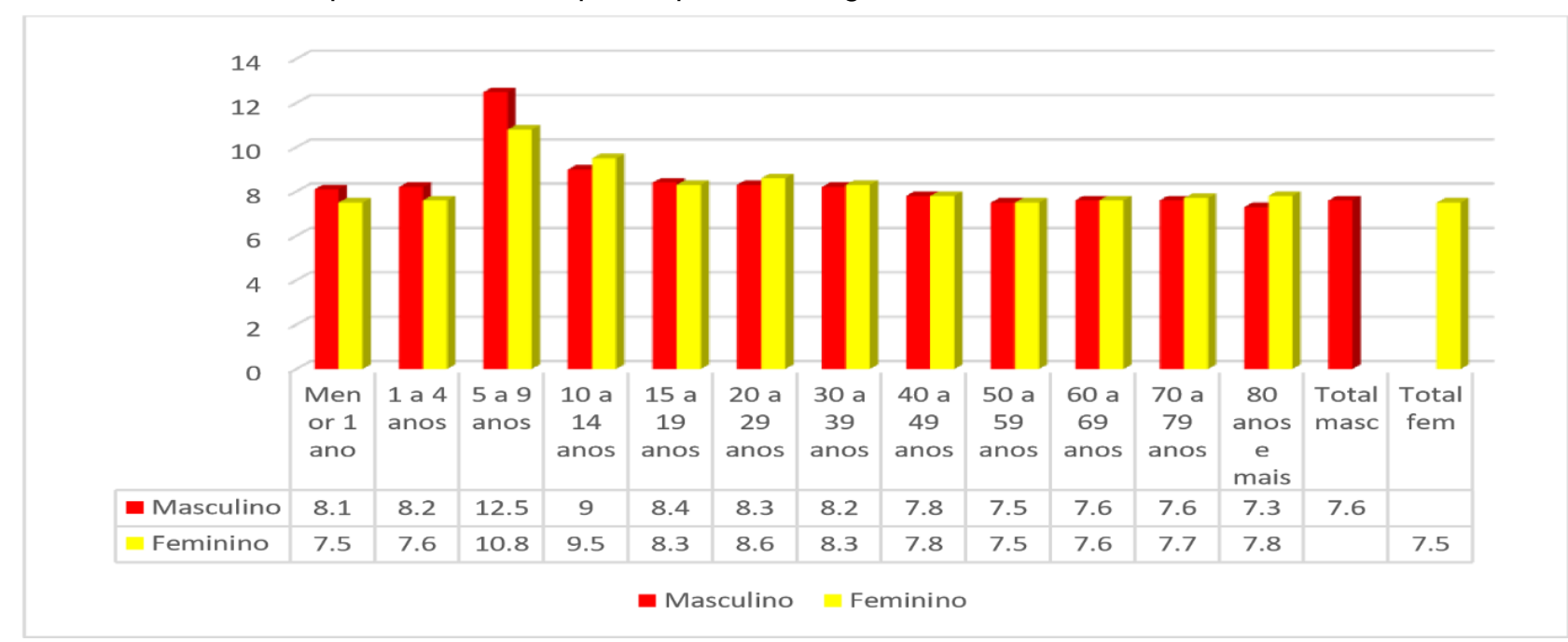

Fonte: Barbosa AML, et al., 2020. Dados extraídos do SIH/DATASUS.

Apesar de André C (2006) afirmar que a raça com maior probabilidade de desenvolver AVC seja a preta, neste estudo observou-se uma maior frequência nos pardos com $46,75 \%$ dos casos, seguido dos brancos com $5,46 \%$ e amarela com $2,22 \%$.

Analisando o Gráfico 3, observa-se uma média de permanência hospitalar total, para o sexo masculino, de cerca de 7,6 dias e menor para o sexo feminino, com 7,5 dias. Em relação à idade, destacou-se, tanto no sexo feminino quanto no masculino, a faixa etária de 5 a 9 anos, com uma média de 10,8 dias de internação das mulheres e 12,8 dias dos homens, apresentando uma média de 11,6 dias considerando-se ambos os sexos. Explicando esses números, Rafay MF (2008) afirma que o AVC é incomum em crianças, fazendo com que os médicos raramente o considerem como primeiro diagnóstico quando surgem os sintomas.

Além disso, para Mekitarian Filho CE e Brunow W (2009), a dificuldade de um diagnóstico adequado em crianças e jovens, considerando que os sinais e sintomas são pouco específicos e podem ser confundidas com outras alterações neurológicas, pode contribuir negativamente no prognóstico do paciente. Outro fator importante, é que apesar de o tempo de permanência hospitalar de adultos e idosos, em comparação com jovens e crianças, ser menor, o índice de mortalidade deles (adultos e idosos) por AVC é superior, como mostra o Gráfico 3.

Segundo Amante LN, et al. (2009), o paciente acometido pelo AVC precisa de cuidados intensivos em algum momento durante sua hospitalização, principalmente na emergência e isso interfere diretamente no tempo de permanência deles nos hospitais. Para Matos JJFC, et al. (2013), no nordeste Brasileiro, apenas uma pequena porcentagem de pacientes consegue chegar ao pronto socorro nas primeiras três horas após o acometimento do AVC, dificultando, assim, o prognóstico favorável do paciente e, consequentemente, aumentando o seu tempo de internação.

Ao analisar os Gráficos 4 e 5, verifica-se que a taxa de mortalidade não sofreu alterações bruscas, tendo uma média de $17,74 \%$, sendo 2012 o ano com a maior taxa (18,53\%) e $2018 \mathrm{com}$ a menor (16,43\%); a idade mais afetada foi com 80 anos ou mais. Embora o gênero masculino tenha maior incidência, constatou-se que o sexo feminino possui maior letalidade com uma taxa de 17,70\%, sendo a faixa etária feminina mais atingida a de 80 anos ou mais. Essa maior mortalidade nas mulheres confirma os dados de Lotufo PA, et al. (2017), que afirma ser o sexo feminino o de maior mortalidade por AVC. 
Gráfico 4 - Taxa de mortalidade por sexo segundo faixa etária

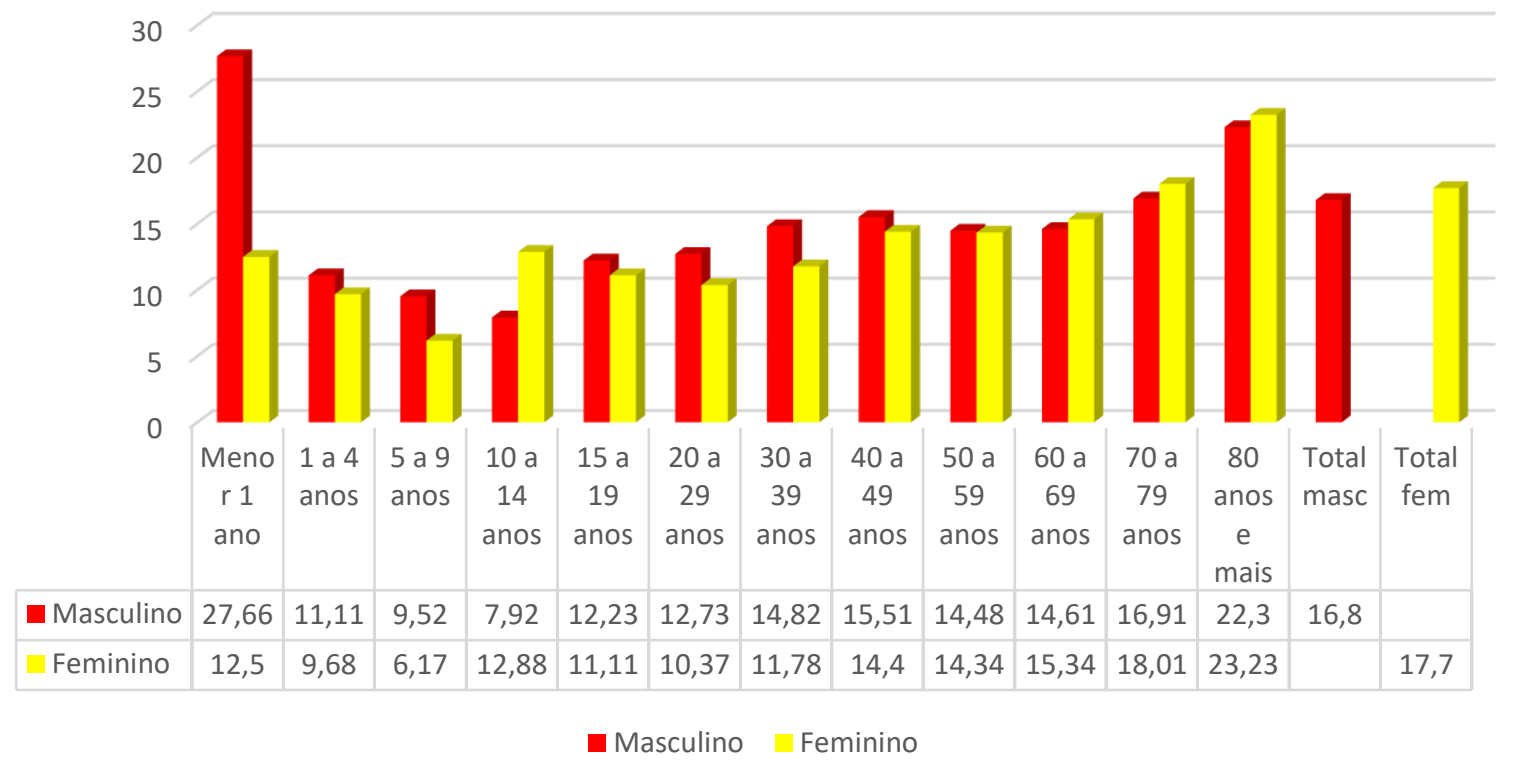

Fonte: Barbosa AML, et al., 2020. Dados extraídos do SIH/DATASUS.

Gráfico 5 - Taxa de mortalidade segundo ano.

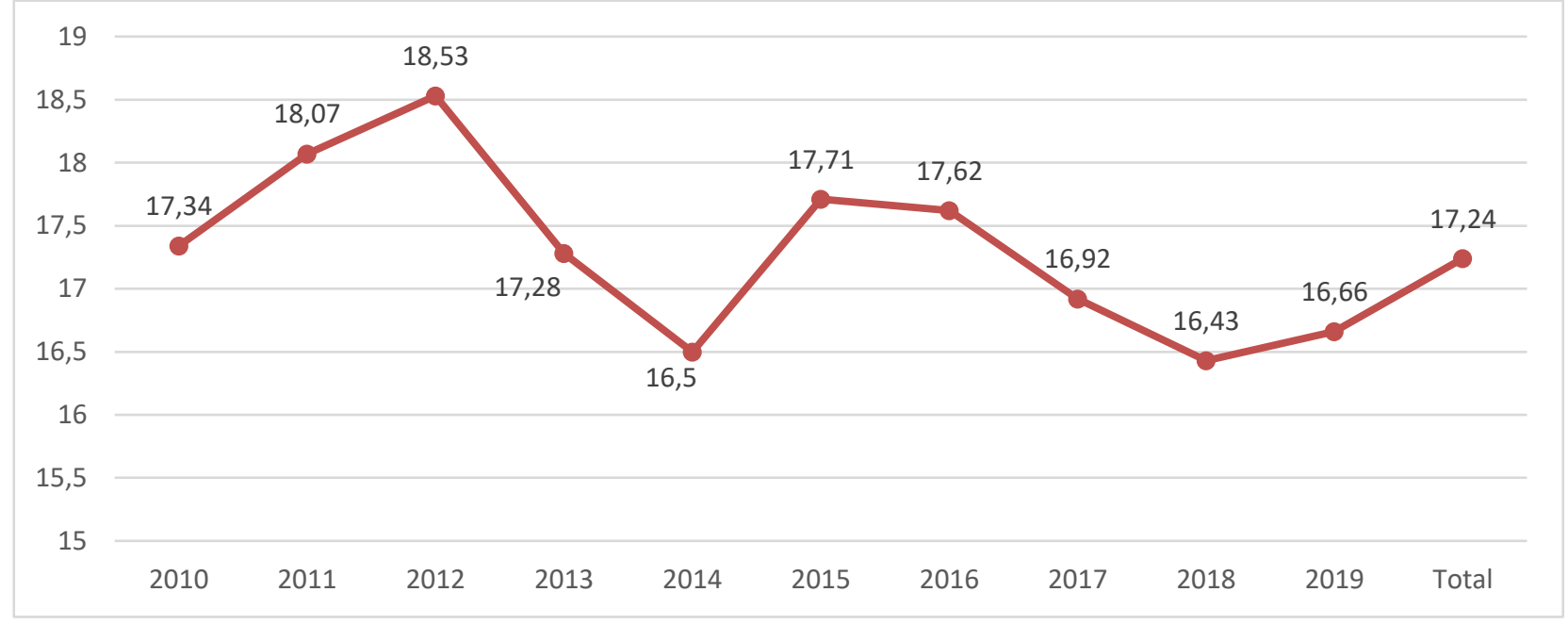

Fonte: Barbosa AML, et al., 2020. Dados extraídos do SIH/DATASUS.

Segundo dados do Ministério da Saúde, o AVC atinge cerca de 16 milhões de pessoas no mundo a cada ano. Dessas, seis milhões morrem. A Organização Mundial de Saúde (OMS) recomenda a adoção de medidas urgentes para prevenção e tratamento da doença. No Brasil, são registradas aproximadamente 68 mil mortes por AVC anualmente. A doença é a principal causa de morte e de incapacidade no país, o que gera um grande impacto econômico para o governo (BRASIL, 2014).

De acordo com o Gráfico 6, independentemente do sexo, os pacientes de 70 a 79 anos foram os mais onerosos. O valor total das internações foi maior no sexo masculino, mais de $R \$ 200$ milhões. Durante os anos analisados, um paciente com AVC custou, para os cofres públicos, uma média de $R \$ 1.080$. Segundo Abramczuk B e Villela E (2009), esse valor pode variar de acordo com a gravidade de cada caso.

Em pacientes que conseguem recuperar completamente o déficit neurológico, necessita-se de um tempo curto de internação ( 3 a 5 dias), a um custo de aproximadamente $R \$ 640$, já nos casos em que o paciente apresenta sequelas mais graves, o tempo de internação pode durar mais de um mês e o custo pode chegar a $\mathrm{R} \$ 32$ mil. 
Gráfico 6 - Valor total das internações por sexo, segundo a faixa etária.

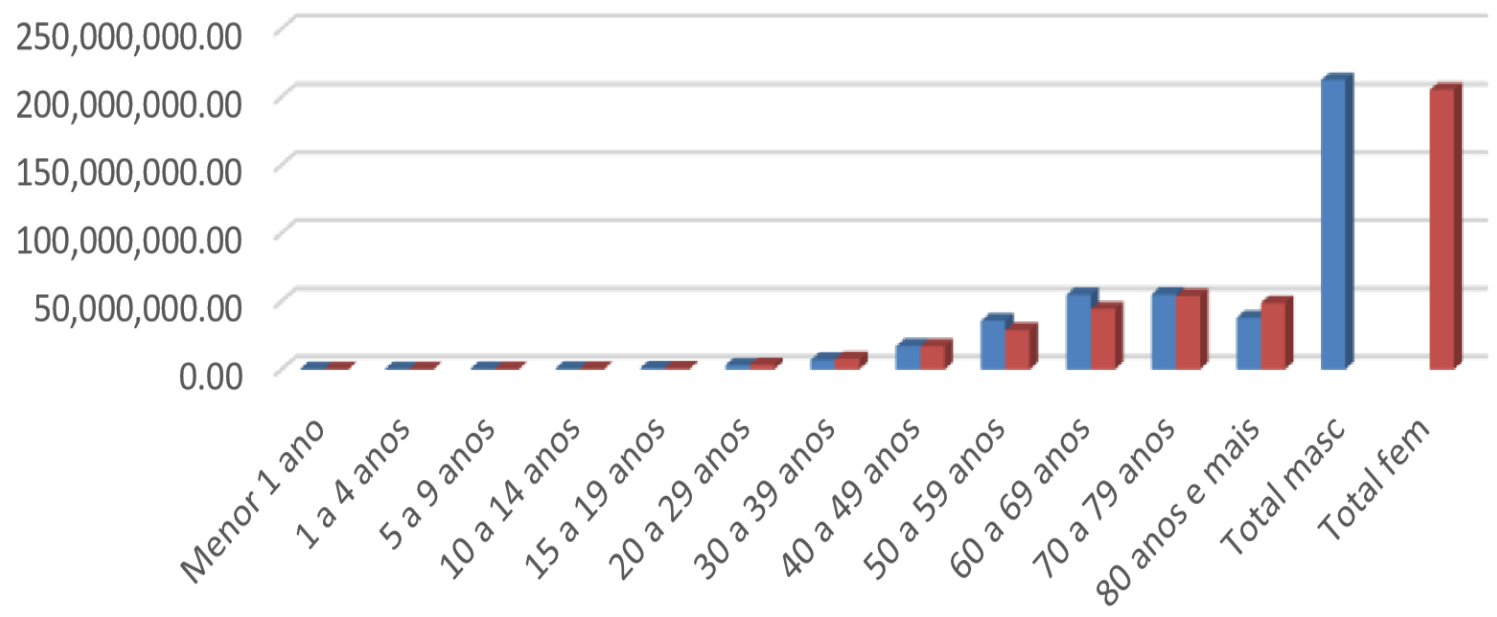

Masculino Feminino

Fonte: Barbosa AML, et al., 2020. Dados extraídos do SIH/DATASUS.

\section{DISCUSSÃO}

A princípio em uma comparação com uma pesquisa realizada por Lopes JM, et al. (2013), com uma metodologia semelhante, mas com diferenças quanto à população da metodologia e ao período analisado, investigou a incidência do AVC isquêmico no Nordeste brasileiro, diferentemente do nosso estudo, foi encontrado uma diminuição nos casos de AVC. Isso comprova a importância do estudo e sua originalidade.

Analisando de relance todos os gráficos, infere-se que a quantidade de internações, durante o período de análise, aumentou gradativamente com o passar dos anos. Com isso, foram estabelecidos parâmetros que compararam esses dados com intuito de analisar os possíveis fatores que estão afetando esse aumento gradual. Esse aumento tem como influência tanto o aumento do envelhecimento da população e a mudança no estilo de vida quanto um maior desenvolvimento tecnológico, o qual facilita a obtenção de diagnósticos, aumentando, assim, o número de casos relatados.

Ademais, mencionando os fatores que influenciam a patologia, de acordo com Araújo APS, et al. (2008), o aumento do número de pacientes acometidos pelo AVC ocorre devido a um maior percentual de pessoas que apresentem os fatores de risco relacionados com sua gênese, como hipertensão arterial, diabetes mellitus, doenças cardíacas, tabagismo e obesidade. Deste modo, percebe-se a importância do controle da morbimortalidade relacionada a essa patologia no Brasil, através, principalmente, de programas do governo, representados centralmente pela Estratégia de Saúde da Família (LIMA V, et al., 2006).

Outrossim, a respeito dos dados fornecidos pelo Gráfico 2, a incidência desta doença está relacionada com idade e expectativa de vida da população, o acelerado crescimento populacional de pessoas idosas, em todo o mundo, aumentará ainda mais as taxas de morbimortalidade dela. Para Wolf CDA (2000) esse crescimento é preocupante em algumas regiões do mundo, como a América Latina e a Ásia, onde se espera um grande aumento na população idosa e essa seria uma das razões que justificariam a estimativa de que a quantidade de AVCs continue aumentando nesses continentes.

Dessa forma, é evidente que o número de internações devido a casos de AVC está intimamente relacionado ao envelhecimento da população, tanto no que diz respeito ao sexo masculino quanto ao sexo feminino. Assim, faz-se necessário a adoção de medidas voltadas para essa faixa etária da população (indivíduos acima de 60 anos), com o intuito de mitigar os fatores de risco controláveis, como a hipertensão arterial, a obesidade, o tabagismo e o etilismo. 
Assim, baseado no Gráfico 3, verifica-se que a média de permanência hospitalar é um critério de grande valia para avaliar o prognóstico dos pacientes, uma vez que um menor o tempo de permanência hospitalar seria um indicativo de um melhor prognóstico. Contudo, apesar da prevalência de permanência ser dos jovens e das crianças, os adultos e idosos acabam por serem os grupos com maior taxa de mortalidade o que pode indicar que o menor tempo de permanência hospitalar desses grupos seja um indicativo de óbito precoce.

Com a mortalidade evidenciada nos gráficos, vale ressaltar uma notável diferença entre nosso estudo e um estudo com dois mil casos realizado pelo British Medical Journal (2005), que aponta pacientes negros sobrevivem com maior frequência que os brancos quando acometidos pelo AVC, porém, nosso estudo observou que a raça com maior taxa de mortalidade foi a parda com $16,06 \%$, seguido da preta com $15,32 \%$ e a branca com $14,89 \%$.

A partir dos Gráficos 4 e 5, uma hipótese que pode justificar as altas taxas de mortalidade por AVC na região nordeste do Brasil é a precariedade de serviços de urgência, como o SAMU, que tenham um suporte avançado na detecção de AVC, a escassez de serviços que apliquem o protocolo de terapia trombolítica e de profissionais treinados. Além disso, o difícil diagnóstico por imagem, assim como a infraestrutura deficitária das redes hospitalares e de seus insumos são fatores que contribuem para a persistência dessa problemática (LOPES JM, et al., 2013).

A sociedade brasileira de neurologia afirma que no Brasil são registrados aproximadamente 100 mil óbitos por ano devido ao AVC, e a cada 5 minutos morre uma pessoa em decorrência desse acometimento (CESÁRIO CMM, et al., 2006). Dessa maneira, constata-se que essa informação abordada pelos autores está em concordância com a alta taxa de mortalidade evidenciada no estudo.

A taxa de mortalidade, dos casos de AVC, é um importante indicativo tanto do aumento de fatores de risco com a idade avançada quanto da preparação do sistema de saúde para tratar essa patologia. Ainda analisando comparativamente o Gráfico 4 e 5, observa-se que a mortalidade é maior em pacientes idosos e que com o passar dos anos essa taxa de mortalidade oscilou, aumentando e diminuindo gradativamente, o que é sugestivo desse embate entre o envelhecimento da população e a melhoria das técnicas de tratamento.

Vale ressaltar que no Gráfico 6, os idosos, com faixa etária superior a 50 anos, necessitaram de maior suporte financeiro para o tratamento da doença. De acordo com Bittencourt SA, et al. (2006), a elevação dos custos da assistência hospitalar aos idosos, em relação à dos mais jovens, está relacionada com o aumento do consumo de procedimentos hospitalares e não com a realização de procedimentos mais caros.

O custo de internações hospitalares é uma variável que está intimamente relacionada ao tempo de permanência hospitalar e ao número de internações totais de cada faixa etária. Esse custo, como evidenciado, é maior na população idosa e menor nos jovens, o que contradiz o tempo de permanência hospitalar que, como foi visto, é maior para jovens e crianças do que para adultos e idosos. Contudo, no que diz respeito ao número absoluto de internações os idosos comandam essa estatística, isso contribui, portanto, para que esse grupo de pacientes sejam os mais onerosos aos cofres públicos.

\section{CONCLUSÃO}

Observou-se, por meio do estudo, que o AVC é um problema frequente na região Nordeste, onde notouse elevada quantidade de casos da doença, com mais de 385 mil casos notificados durante o período analisado, o que evidencia a necessidade de investimentos nas políticas de suporte e prevenção dessa patologia. No estudo realizado, mostrou-se a predominância do sexo masculino entre os casos de AVC mais notificados no Nordeste do Brasil. A principal faixa etária acometida, em ambos os sexos, foi entre 70 e 79 anos e é possível perceber que, no decorrer deste tempo, a incidência da doença não sofreu grandes variações, mantendo-se com um grande número de casos. A morbidade hospitalar, bem como o índice de mortalidade mostrou-se elevada em pacientes com idade superior aos 80 anos, assim como o custo total de internações nessa mesma faixa etária. 


\section{REFERÊNCIAS}

1. ABRAMCZUK B, VILLELA E. A luta contra o AVC no Brasil. Com Ciência, 2009; 109.

2. ALVES HCBR, et al. Vasos sanguíneos colaterais no AVC isquêmico agudo: uma janela fisiológica para prever resultados futuros. Arq. Neuro-Psiquiatr., 2016; 74(8): 662-670.

3. AMANTE LN, et al. Nursing care systematic zati on at the Intensive Care Unit (ICU) based on Wanda Horta's theory. Revista Escola de Enfermagem USP, 2009; 9(13): 55-59.

4. ANDRÉ C. Manual de AVC. 2 ed. Rio de Janeiro: Revinter, 2006.

5. ARAÚJO APS, et al. Prevalência dos fatores de risco em pacientes com acidente vascular encefálico atendidos no setor de neurologia da clínica de fisioterapia da UNIPAR, campus sede. Arquivo. Ciência. Saúde Unipar, 2008; 12(1): 35-42.

6. BERGAMASCHI DP, HINNIG P. FSP/USP. HEP139 - Informática/Nutrição - 2011. British Medical Journal, 2005; $331: 431$.

7. BITTENCOURT SA, et al. O Sistema de Informação Hospitalar e sua aplicação na saúde coletiva. Cadernos de Saúde Pública, 2006; 22(1): 19-30.

8. BRASIL. Ministério da Saúde. Acidente Vascular Cerebral (AVC), 2014.

9. CESÁRIO CMM, et al. Impacto da disfunção motora na qualidade de vida em pacientes com Acidente Vascular Encefálico. Revista Neurociências, 2006; 14(1): 6-9.

10. FERREIRA LE, et al. Biobanco de AVE em Joinville: protocolo de estudo e resultados do primeiro ano. Arq. NeuroPsiquiatr., 2017; 75(12): 881-889.

11. FURLAN NE, et al. Association between blood pressure and acute phase stroke case fatality rate: a prospective cohort study. Arq. Neuro Psiquiatr., 2018; 76(7): 436-443.

12. LIMA V, et al. Fatores de risco associados à hipertensão arterial sistêmica em vítimas de acidente vascular cerebral. Revista Brasileira em Promoção a Saúde. 1993 (3): 148-154, 2006.

13. LOPES JM, et al. Acidente vascular cerebral isquêmico no Nordeste brasileiro: uma análise temporal de 13 anos de casos de hospitalização. ConScientiae Saúde, 2013;12(2):321-328.

14. LOTUFO PA, et al. Doença cerebrovascular no Brasil de 1990 a 2015: Global Burden of Disease 2015. Revista Brasileira de Epidemiologia, 2017; 20: 129-141.

15. LUNA ACP, et al. Avaliação da capacidade funcional em pacientes internados em uma unidade de AVC isquêmico no interior do ceará utilizando as escalas de Barthel e Rankin. In: Congresso brasileiro de doenças cerebrovasculares. Anais de epidemiologia, 2013.

16. MAMED SN, et al. Perfil dos óbitos por acidente vascular cerebral não especificado após investigação de códigos garbage em 60 cidades do Brasil, 2017. Rev. bras. epidemiol., 2019; 22(supl. 3): e190013.

17. MATOS JJFC, et al. Perfil de gravidade dos pacientes admitidos em uma unidade de acidente vascular cerebral (AVC) na cidade de fortaleza (CE): uma análise com base na escala do Nihss. Unichristus, Fortaleza, CE. In: Congresso brasileiro de doenças cerebrovasculares. Anais de epidemiologia, 2013.

18. MEKITARIAN FILHO CE, BRUNOW W. Acidentes vasculares encefálicos em pediatria. J. Pediatr. (Rio J.) [online], 2009; 85(6): 469-479.

19. NITRINI R, et al. A neurologia que todo médico deve saber, 3ำ edição. Atheneu, 2015.

20. PIMENTEL BN, FILHA VAVS. Avaliação das funções vestibulares e oculomotoras em indivíduos com tontura após acidente vascular cerebral. Arq. Neuro-Psiquiatr., 2019; 77(1): 25-32.

21. RAFAY MF. Ischaemic stroke in children: overview including an asian perspective. JPak Med. Assoc., 2008; 58(7): 384-90.

22. ROLIM AM, et al. Associação estatística do polimorfismo rs2243250 do gene IL4 e acidente vascular cerebral hemorrágico na população brasileira. J. Bras. Patol. Med. Lab., 2020; 56: e1872020.

23. RUIZ L, et al. Complicações neurológicas e extra-neurológicas em pacientes com AVC internados no Hospital de Clínicas de Montevidéu por um período de 2 anos. Anfamed, 2020; 7(1): 01209.

24. WOLF CDA. The impact of stroke. Br Med Bull, 2000; 6: 256-286. 